\title{
Polimorfismos de los genes CAPN1, CAST, DES, PRKAG3 y RYR1 asociados a la capacidad de retención de agua en crudo y cocinado en carne de bovino en cruces Bos indicus y Bos taurus en Colombia
}

\author{
Leal-Gutiérrez, J.D. ${ }^{1 @ ; ~ J i m e ́ n e z-R o b a y o, ~ L . M . ~}{ }^{1}$; Ariza, M. ${ }^{2}$; Manrique, C. ${ }^{2}$; López, J. ${ }^{2}$; Martínez, C. ${ }^{2}$; Pinilla, Y. ${ }^{1}$; Castro, S. ${ }^{2}$; \\ García, N. ${ }^{2}$; Bedoya, C. ${ }^{2}$ y Jiménez, A. ${ }^{3}$
}

\begin{abstract}
'Unidad de Genotipificación de Animales Domésticos (UGA). Departamento de Ciencias de la Producción Animal. Facultad de Medicina Veterinaria y de Zootecnia. Universidad Nacional de Colombia. Bogotá. Colombia.

2Departamento de Ciencias de la Producción Animal. Facultad de Medicina Veterinaria y de Zootecnia. Universidad Nacional de Colombia. Bogotá. Colombia.

${ }^{3}$ Asociación Colombiana de Criadores de Ganado Cebú. ASOCEBÚ. Colombia.
\end{abstract}

\section{Palabras ClaVe adicIONALES}

Marcador genético.

Mutación.

Parámetros de calidad.

Producto final.

\section{RESUMEN}

La capacidad de retención de agua (CRA) es uno de los parámetros de calidad de la carne más importante, dado que posee asociación con la percepción de jugosidad y la pérdida de peso de la pieza cárnica durante procedimientos como la maduración, la cocción y otro tipo de procesamiento tecnológico. La CRA en crudo y cocinado (CRAr y CRAc respectivamente) fue evaluada en 164 machos castrados cruzados, en los cuales se realizó la genotipificación de 23 regiones genómicas de los genes CAPN1, CAST, DES, PRKAG3 y RYR 1. El marcador CAST2959 presentó asociación con el parámetro CRAr en el músculo Longissimus dorsi (LD) siendo el genotipo AA el de mejor comportamiento. El marcador RYR 1-1 1195 se encontró asociado con el parámetro CRAc en LD, siendo la carne de animales con los genotipos GG y GA, la de mayor pérdida de peso, si son comparados con carne proveniente de animales con genotipo AA. Lo anterior permite establecer estos marcadores como posibles polimorfismos de importancia en procesos de selección y mejoramiento animal de este parámetro de calidad cárnica (CRAr y CRAc) en bovinos, posterior a un proceso de validación.

\section{Polymorphisms of CAPN1, CAST, DES, PRKAG3 and RYR1 genes associated with water holding capacity in raw and cooked meat of crossbred bovine Bos indicus and Bos taurus in Colombia}

\section{SUMMARY}

Water holding capacity (WHC) is a more important quality trait in meat and is associated with juiciness and weight loss of meat cutting during technologic procedures like aging, cooking, and thawing. WHC in raw (WHCr) and cooked (WHCc) meat were assessed in 164 cross-castrated bulls. These animals were genotyped in 23 genomic regions in CAPN1, CAST, DES, PRKAG3 and RYR1 genes. CAST2959 polymorphism was established as associated with WHCr in Longissimus dorsi (LD) and animals with genotype AA had the desired parameter. RYR 1-1 1195 marker had association with WHCc in LD and steaks of animals with genotypes GG and GA had bigger weight loss than animals AA. Thus, these polymorphisms would be useful in animal selection and breeding programs involved in this meat feature (WHCr and $\mathrm{WHCc}$ ) after a validation process.

\section{INFORMACIÓN}

Cronología del artículo:

Recibido/Received: 24-4-2014

Aceptado/Accepted: 22-1-2015

On-line: 16.3 .2015

Correspondencia a los autores/Contact e-mail:

jdlealg@unal.edu.co

\section{INTRODUCCIÓN}

Todas las características asociadas al desarrollo de tejidos del cuerpo están sujetas a un gobierno genético y en producción animal se han establecido mutaciones en genes mayores con amplia influencia en parámetros asociados al contenido de grasa y agua en el músculo
(Enfält et al., 2006) y por lo tanto con parámetros de calidad del producto final (Škrlep et al., 2010a; Škrlep et al., 2010b; Lindahl et al., 2004a; Lindahl et al., 2004b; Lahucky et al., 1997; Otto et al., 2007). Este es el caso de los marcadores $\mathrm{rn}+, \mathrm{rn}^{*}$ y RN- del gen PRKAG3 del porcino, los cuales han conducido los criterios de selección de varias razas de esta especie doméstica. Los 
parámetros asociados a la capacidad de retención de agua (CRA) de la carne no escapan a este tipo de control ni al efecto de los polimorfismos en genes mayores asociados (Lindahl et al., 2004a y 2004b).

La característica de calidad cárnica, CRA puede ser considerada de alta importancia productiva y económica, dada la pérdida de peso de la carne que ocurre durante el almacenamiento y la cocción, lo que influye en la percepción de calidad del consumidor. El primer parámetro a considerar para su evaluación subjetiva, es la CRA en crudo (CRAr), la cual evoluciona según el desarrollo de una estructura proteica más homogénea durante la maduración de la carne en el post-mortem y está determinada por la degradación de proteínas citoesqueléticas del miocito (Straadt et al., 2007). El segundo parámetro se denomina CRA en cocinado (CRAc), que consiste en la expulsión del agua propia de la carne, sumada a la fusión de grasas y pérdida de proteínas solubles (Barbanti y Pasquini, 2005). Lo anterior ocurre como resultado de la contracción de las miofibrillas, en respuesta a la denaturación proteica generada por la temperatura de cocción (Straadt et al., 2007). Esta denaturación genera la exposición de regiones hidrofóbicas de la estructura miofibrilar y conduce a nuevas interacciones intra e intermoléculares originando una estructura proteica más densa (Straadt et al., 2007).

Las características de calidad cárnica en bovino se ven afectadas por varios procesos con gobierno genético y pueden ser divididos en dos grupos principales. En el primer grupo se encuentran los genes con influencia sobre la tasa de degradación estructural del miocito la cual es realizada principalmente por el sistema proteolítico de la $\mu$-calpaína y la calpastatina (CAPN1 y CAST respectivamente), la cual conduce a una interacción más estable entre la matriz proteica y el agua, disminuyendo las pérdidas de líquido de la carne. Una de las proteínas de mayor importancia de la matriz proteica es la Desmina (DES), la cual es considerada como el mayor sustrato del sistema proteolítico CAPN1-CAST (Zhang et al., 2006; Bee et al., 2007). El segundo grupo de genes está determinado por aquellos cuya proteína interviene o altera procesos de modulación energética del miocito. Entre ellas se destacan la subunidad gamma 3 de la proteína quinasa AMPK activada (PRKAG3) y el receptor de la rianodina tipo 1. La primera corresponde a la subunidad reguladora de la Proteína Quinasa AMP activada (AMPK) la cual es un sensor de la energía celular, siendo ésta un heterotetrámero sensible al incremento de la concentración de $\mathrm{AMP}$, en respuesta a una disminución en el nivel energético de la célula, a estrés o a un incremento en la tasa AMP:ATP (Grahame et al., 2005). La segunda proteína corresponde al Receptor de la Rianodina tipo I, la cual aunque no es una proteína energética, está encargada de la regulación de la contracción-relajación del músculo esquelético mediante el control de la liberación de $\mathrm{Ca}^{2+}$ desde el retículo sarcoplásmico, pudiendo afectar el metabolismo energético celular al conducir el consumo de ATP (Felder et al., 2002). Cualquier alteración en los procesos relacionados con el metabolismo de ATP pueden conducir a una modificación en la tasa de con- versión de glicógeno a ácido láctico, lo que modula el descenso del pH del músculo-carne en el post-mortem.

El objetivo de este estudio fue establecer la existencia de polimorfismos de nucleótido simple (SNPs) en los genes CANP1, CAST, DES, PRKAG3 y RYR1 en la población analizada y determinar la asociación de estas mutaciones con los parámetros capacidad de retención de agua en carne cruda (CRAr) y cocida (CRAc).

\section{MATERIAL Y MÉTODOS}

\section{POBLACIÓN ANALIZADA}

Se analizaron 164 machos castrados F1 provenientes del cruce entre hembras Brahman Blanco con reproductores de las razas Limousin, Normando, Braunvieh, Simental, BON, Romosinuano (Bos taurus), Guzerat, Brahman Rojo y Brahman Blanco (Bos indicus), los cuales fueron distribuidos en un total de ocho lotes de engorde en dos fincas del sur del Departamento de Cesar, Colombia. Cinco lotes fueron mantenidos en la finca Cabezas y tres lotes en la finca Santa Helena. Los animales fueron alimentados con una dieta a base de los forrajes brachipará (Brachiaria plantaginea), guinea (Panicum maximum) y ángleton (Dichantium aristatum) y un suplemento mineral (Martínez et al., 2012). El sacrificio fue realizado a un peso promedio de $492 \pm 38.5$ $\mathrm{kg}$ y una edad promedio de 30,9 $9 \pm 4,15$ meses (la tabla I presenta los valores promedio/cruce) en una planta de beneficio (La Dorada, Caldas, Colombia) mediante procedimientos comerciales estándar. La población utilizada es la misma empleada en Leal et al. (2014).

\section{OBTENCIÓN DE LOS PARÁMETROS FENOTÍPICOS}

Los músculos Longissimus dorsi (LD) y Semitendinosus (ST) fueron extraídos de la canal izquierda, empacados al vacio y transportados a las instalaciones del Instituto de Ciencia y Tecnología de Alimentos ICTA (Universidad Nacional de Colombia). Cada músculo fue dividido en tres porciones, una para cada tiempo de maduración $(7,14$ y 21 días) y posteriormente cada porción fue dividida en dos piezas cárnicas que fueron sometidas al proceso de maduración en un refrigerador industrial $\left(4^{\circ} \mathrm{C}\right)$. La pieza 1 fue utilizada para la medición de parámetros en carne cruda y la 2 para

Tabla I. Valores promedio y desviaciones estándar para el peso y la edad de sacrificio de los animales según cruce (Means and standard deviation of slaughter weight and age by cross).

\begin{tabular}{lccccc}
\hline \multirow{2}{*}{ Cruce } & \multirow{n}{c}{} & \multicolumn{2}{c}{ Peso $(\mathrm{kg})$} & \multicolumn{2}{c}{ Edad (meses) } \\
\cline { 3 - 6 } & & Promedio & SE & Promedio & SE \\
\hline BON & 18 & 501,11 & 51,43 & 26,67 & 4,55 \\
Br Blanco & 44 & 497,91 & 35,62 & 27,75 & 4,14 \\
Br Rojo & 10 & 495,40 & 47,36 & 27,85 & 4,71 \\
Braunvieh & 8 & 486,25 & 35,50 & 25,10 & 3,28 \\
Guzerat & 17 & 483,75 & 29,73 & 27,99 & 5,30 \\
Limousin & 20 & 479,10 & 28,23 & 24,21 & 3,09 \\
Normando & 17 & 515,35 & 28,22 & 25,98 & 3,59 \\
Romosinuano & 12 & 485,75 & 31,09 & 25,10 & 1,93 \\
Simental & 18 & 511,72 & 39,71 & 25,07 & 3,99 \\
\hline
\end{tabular}


Tabla II. Ubicación de los polimorfismos encontrados en la población analizada (Polymorphisms found in the assessed population, and its locations).

\begin{tabular}{|c|c|c|c|c|}
\hline Nombre del SNP & Nucleótido & Secuencia contenedora & Ubicación* & Reporte \\
\hline CAPN 5331 & 161 & GAGGGCGGGG(A/T)GGAGCGCTCT & 11 & Casas et al., 2005 \\
\hline CAPN 316 & 5544 & GAGTGGAACG(C/G)CGTGGACCCT & E 9 & Page et al., 2002 \\
\hline CAPN 530 & 22128 & GGATGACCAG(A/G)TCCAGGCCAA & E 14 & Page et al., 2002 \\
\hline CAPN 4751 & 24114 & GGGGGGAAAA(T/C)CGAGGCGCAG & | 17 & White et al., 2005 \\
\hline UoGCAST & 84719 & TTCAAATTTT(G/C)TACCCAAAGT & 18 & Schenkel et al., 2006 \\
\hline CAST 1 & 85920 & AGAAGGACTT(T/C)TTCGTTATGA & 19 & Chung et al., 2001 \\
\hline CAST 2870 & 134877 & TAGTATTTTC(A/G)ATTTGCACTT & E 32 & Corva et al., 2007 \\
\hline CAST 2959 & 134966 & СTTTCCTCTT(G/A)GACTTGTGGA & E 32 & Barendse et al., 2007 \\
\hline PRKAG3-1526 & 1526 & CAGCCTCCСС(G/T)CTGACTGTAC & E 4 & Yu et al., 2005 \\
\hline PRKAG3-2180 & 2180 & AAACAAGGTA(C/T)ATCATTTTGA & 14 & Yu et al., 2005 \\
\hline PRKAG3-2745 & 2745 & AAGCAGAGCTT(C/T)GTGGGTGAGG & E 5 & $\mathrm{NCBI}$ \\
\hline PRKAG3-2925 & 2925 & GGTCCAGGGT(A/G)AACCCCACTC & 15 & No reportado \\
\hline PRKAG3-2961 & 2961 & CCCACCCCCC(A/G)CAGGGATGCT & 15 & No reportado \\
\hline PRKAG3-3047 & 3047 & TCAGGGTCCT(A/G)GGGGCACCCA & 16 & No reportado \\
\hline PRKAG3-3068 & 3068 & TCTGGACTGG(A/G)GCGGAAGGAG & 16 & Yu et al., 2005 \\
\hline PRKAG3-3078 & 3078 & GGCGGAAGGA(A/G)TTCAGGAAGC & 16 & No reportado \\
\hline PRKAG3-5898 & 5898 & СTCCTGGGTT(G/A)GTGTCССTCT & E 13 & $\mathrm{NCBI}$ \\
\hline RYR1-11195 & 11195 & ATGCTGCCCC(G/A)GACCCCAAGG & E 11 & $\mathrm{NCBI}$ \\
\hline RYR1-13638 & 13638 & ACATGGACGA(T/C)GCACTGTCAC & E 12 & $\mathrm{NCBI}$ \\
\hline RYR1-20298 & 20298 & АGCTTCTGCT(G/A)CAGACAAACC & E 17 & $\mathrm{NCBI}$ \\
\hline RYR1-21643 & 21643 & TCAGCTGCTG(T/C)CTGGACCTCA & E 19 & $\mathrm{NCBI}$ \\
\hline RYR1-26680 & 26680 & TGGGCATGGC(C/A)GATGAGAAGG & E 23 & $\mathrm{NCBI}$ \\
\hline RYR1-27871 & 27871 & TCCCTGCCCG(C/T)CGGAACCCTC & E 24 & $\mathrm{NCBI}$ \\
\hline RYR1-27970 & 27970 & TGCTGGGCTA(T/C)GGCTACAACA & E 24 & $\mathrm{NCBI}$ \\
\hline RYR1-27982 & 27982 & TGCTGGGCTA(T/C)GGCTACAACA & E 24 & $\mathrm{NCBI}$ \\
\hline RYR1-28046 & 28046 & ССCTGGACTC(G/C)AATCTCTGAC & 124 & No reportado \\
\hline RYR1-28068 & 28068 & CAACTCAGCC(T/C)CTCAGTGGGC & 124 & No reportado \\
\hline
\end{tabular}

*Ubicación del polimorfismo I= intrón y E= exón.

carne en cocinado. Una vez se cumplían los tiempos de maduración mencionados cada pieza cárnica era dividida en tres lonchas de 2,54 cm de grosor (Chung y Davis, 2011); cabe destacar que en cada una de las lonchas provenientes de la pieza 1 se midió el parámetro CRAr y en cada una de las de la pieza 2 el parámetro CRAc. Cuando se cumplía el respectivo tiempo de maduración de la pieza 2, ésta era sometida a congelación $\left(-20{ }^{\circ} \mathrm{C}\right)$ hasta su procesamiento y el loncheado era realizado con sierra eléctrica.

La CRAr se determinó usando la metodología de F.p.p. de Grau y Hamm modificada descrita por Van Oeckel et al. (1999), aplicando 2,2 kg de fuerza por $5 \mathrm{mi}-$ nutos a una muestra de $400 \mathrm{mg}$ de carne molida, sobre papel Walkman $\mathrm{n}^{\circ} 5$ con un texturómetro TA-XT plus (Texture Analyser Stable Micro-Systems) y el software Sponent 32. Como resultado se producen dos áreas en el papel filtro, una denominada $\mathrm{M}$ que determina el valor de la carne aplanada y la otra T que corresponde al área dejada por el agua expulsada. El porcentaje de CRAr se determinó por la relación $\mathrm{M}^{*} 100 / \mathrm{T}$, siendo el valor óptimo el más elevado. La CRAc fue evaluada mediante la cocción en asador de doble contacto a una temperatura de $70{ }^{\circ} \mathrm{C}$ en centro geométrico y calculada utilizando la metodología de Bertram et al. (2003) mediante la ecuación: CRAc $=100$ * ((Peso crudo-Peso cocinado)/Peso crudo). Leal et al. (2014) presentan el comportamiento de los parámetros asociados a la ca- pacidad de retención de agua en crudo y en cocinado según el tipo racial y el tiempo de maduración utilizando la misma población.

\section{GENOTIPIFICACIÓN DE LA POBLACIÓN}

Las reacciones de PCR fueron realizadas a un volumen final de $25 \mu \mathrm{l}$, consistente en $8,26 \mu \mathrm{l}$ de agua ultrapura para PCR, $2,5 \mu \mathrm{lde} \mathrm{MgCl}^{+2}(25 \mathrm{mM}), 5 \mu \mathrm{l}$ of dNTP ( 2,5 moles de cada desoxiribonucleótido en buffer $5 X)$, $2 \mu \mathrm{l}$ de cada primer $(10 \mathrm{pmol} / \mu \mathrm{l}), 0,24 \mu \mathrm{l}$ de TaqDNA polimerasa $(5 \mathrm{U} / \mu \mathrm{l})$ y $5 \mu \mathrm{l}$ de templete de $\mathrm{ADN}$ extraído a partir de sangre o tejido muscular $(50 \mathrm{ng} / \mu \mathrm{l})$. Los polimorfismos fueron hallados en la población analizada utilizando las técnicas RFLP ó SSCP (Bastos et al., 2001; Sunnucks et al., 2000; Cui et al., 2006). La región analizada en el gen DES correspondió a la amplificada por los cebadores F:TACAAATAGTGCGGACGGCCGGGGG y R:GGTGCGGCGGTAGGACGACACCCGC, la cual fue determinada como no informativa. Posterior a la genotipificación de los marcadores, se realizó su secuenciación, lectura de electroferogramas y determinación de la posición polimórfica (tabla II).

\section{ANÁLISIS ESTADÍSTICO}

Las frecuencias genotípicas y alélicas de los polimorfismos fueron determinados mediante el procedimiento PROC FREQ de SAS. En el análisis de asociación genotipo-fenotipo solo fueron incluidos marcadores con una frecuencia en su alelo menor, superior al 
$10 \%$ (Lee et al., 2011; Curi et al., 2010). Adicionalmente, los genotipos con número reducido de animales fueron excluidos para prevenir una influencia negativa en la estimación de medias (Café et al., 2010; Corva et al., 2007), empleando como limite 8 individuos. Para el análisis asociación genotipo-fenotipo se utilizó el procedimiento GLM de de SAS (Reardon et al., 2010; Otto et al., 2007; Zhou et al., 2010). El modelo aplicado fue el siguiente:

$$
\begin{gathered}
\text { Yijklmn }=\mu+P i+S j+M k+P i^{*} S j+P i^{*} M k+S j^{*} M k \\
+P i^{*} S j^{*} M k+G l+A m(G l)+e i j k l m n
\end{gathered}
$$

Donde:

Yijklmn= Variable CRA.

$\mu=$ Media poblacional.

$\mathrm{Pi}=$ Efecto del genotipo i-ésimo del marcador analizado (dos a tres niveles según el marcador).

$\mathrm{S} \mathbf{j}=$ Efecto del grupo racial j-ésimo (dos niveles; Bos taurus*Bos indicus y Bos indicus*Bos indicus).

Mk= Efecto del tiempo de maduración k-ésimo (tres niveles).

$\mathrm{Pi}^{*} \mathrm{Sj}=$ interacción genotipo*grupo racial.

$\mathrm{Pi}^{*} \mathrm{Mk}=$ interacción genotipo*tiempo de maduración.

$\mathrm{Si}^{*} \mathrm{Mj}$ = interacción grupo racial*tiempo de maduración.

$\mathrm{Pi}^{*} \mathrm{Sj}^{*} \mathrm{Mk}=$ interacción marcador ${ }^{*}$ grupo racial ${ }^{*}$ tiempo de maduración.

Gl= Efecto del grupo contemporáneo l-ésimo (ocho niveles; animales de igual procedencia y día de sacrificio).

$\operatorname{Am}(\mathrm{Gl})=$ Animal m-ésimo anidado dentro del grupo de contemporáneo l-ésimo.

Eijklmn= Error aleatorio.

El modelo estadístico fue aplicado por cada marcador analizado (Otto et al., 2007; Brunner et al., 2012). Los factores fueron determinados como efectos fijos (Škrlep et al., 2010; Otto et al., 2007; Casas et al., 2005). Para comprobar la significancia del factor genotipo se empleó el anidamiento Am(Gl) como error. Se aplicó la corrección de Bonferroni $(\alpha=\alpha / n)$ por cada músculo. Para los marcadores que se encontraron significativos se aplicaron las comparaciones de Tukey-Kramer.

\section{RESULTADOS}

En la tabla III se presentan las frecuencias alélicas y genotípicas de los marcadores evaluados. Únicamente se encontró asociación fenotipo-genotipo para el músculo LD. El polimorfismo CAST2959 presentó asociación con el parámetro CRAr (tabla IV), siendo el genotipo benéfico AA. Para el parámetro CRAc se presentó asociación con el marcador RYR1-11195, siendo las piezas cárnicas de animales con genotipo AA, aquellas con menores pérdidas de peso durante la cocción.

\section{DISCUSIÓN}

\section{Genes del sistema CAPN1-CAST}

En el presente estudio el marcador CAST2959 se estableció como el único polimorfismo del sistema CAPN1-CAST evaluado que presentó significancia en la asociación con el parámetro CRAr en el músculo LD, siendo los individuos AA aquellos con el mejor desempeño respecto a animales AG y GG. De modo similar
Tabla III. Frecuencias alélicas y genotípicas en cada

\begin{tabular}{|c|c|c|c|c|c|}
\hline \multirow{2}{*}{$\begin{array}{l}\text { Marcador } \\
\text { CAPN316* }\end{array}$} & \multicolumn{2}{|c|}{ Alelos } & \multicolumn{3}{|c|}{ Genotipos } \\
\hline & C & G & $\mathrm{CC}$ & GC & GG \\
\hline & 0,05 & 0,95 & 0 & 0,09 & 0,91 \\
\hline \multirow{2}{*}{ CAPN530 } & A & G & AA & GA & GG \\
\hline & 0,20 & 0,80 & 0 & 0,40 & 0,60 \\
\hline \multirow{2}{*}{ CAPN4751 } & $\mathrm{C}$ & $\mathrm{T}$ & $\mathrm{CC}$ & $\mathrm{CT}$ & TT \\
\hline & 0,12 & 0,88 & 0,02 & 0,20 & 0,78 \\
\hline \multirow{2}{*}{ CAPN5331 } & $\mathrm{A}$ & $\mathrm{T}$ & $\mathrm{AA}$ & AT & TT \\
\hline & 0,28 & 0,72 & 0,01 & 0,53 & 0,46 \\
\hline \multirow{2}{*}{ CAST1 } & $C$ & $\mathrm{~T}$ & $\mathrm{CC}$ & $\mathrm{CT}$ & $\mathrm{TT}$ \\
\hline & 0,63 & 0,37 & 0,46 & 0,34 & 0,20 \\
\hline \multirow{2}{*}{ CAST2870 } & A & G & AA & AG & GG \\
\hline & 0,40 & 0,60 & 0,13 & 0,53 & 0,34 \\
\hline \multirow{2}{*}{ CAST2959 } & A & G & AA & AG & GG \\
\hline & 0,65 & 0,35 & 0,45 & 0,41 & 0,14 \\
\hline \multirow{2}{*}{ UoGCAST } & C & G & $\mathrm{CC}$ & GC & GG \\
\hline & 0,68 & 0,32 & 0,43 & 0,50 & 0,07 \\
\hline \multirow{2}{*}{ PRKAG3-1526* } & G & $\mathrm{T}$ & GG & TG & $\mathrm{TT}$ \\
\hline & 0,93 & 0,07 & 0,88 & 0,11 & 0,01 \\
\hline \multirow{2}{*}{ PRKAG3-2180 } & $C$ & $\mathrm{~T}$ & $\mathrm{CC}$ & $\mathrm{TC}$ & $\mathrm{TT}$ \\
\hline & 0,16 & 0,84 & 0,01 & 0,32 & 0,68 \\
\hline \multirow{2}{*}{ PRKAG3-2745 } & $\mathrm{C}$ & $\mathrm{T}$ & $\mathrm{CC}$ & $\mathrm{CT}$ & $\mathrm{TT}$ \\
\hline & 0,36 & 0,64 & 0,15 & 0,41 & 0,44 \\
\hline \multirow{2}{*}{ PRKAG3-2925 } & A & G & AA & AG & GG \\
\hline & 0,53 & 0,47 & 0,32 & 0,41 & 0,26 \\
\hline \multirow{2}{*}{ PRKAG3-2961 } & A & G & $\mathrm{AA}$ & $A G$ & GG \\
\hline & 0,62 & 0,38 & 0,32 & 0,59 & 0,09 \\
\hline \multirow{2}{*}{ PRKAG3-3047 } & A & G & AA & $A G$ & GG \\
\hline & 0,38 & 0,62 & 0,09 & 0,59 & 0,32 \\
\hline \multirow{2}{*}{ PRKAG3-3068 } & $A$ & G & AA & $A G$ & GG \\
\hline & 0,22 & 0,78 & 0,03 & 0,38 & 0,59 \\
\hline \multirow{2}{*}{ PRKAG3-3078 } & A & G & AA & $A G$ & GG \\
\hline & 0,25 & 0,75 & 0 & 0,50 & 0,50 \\
\hline \multirow{2}{*}{ PRKAG3-5898 } & A & G & AA & GA & GG \\
\hline & 0,39 & 0,61 & 0,09 & 0,59 & 0,32 \\
\hline RYR1-11195 & A & G & AA & $A G$ & GG \\
\hline राला-11190 & 0,49 & 0,51 & 0,43 & 0,11 & 0,46 \\
\hline RYR1-13638 & $\mathrm{C}$ & $\mathrm{T}$ & $\mathrm{CC}$ & $\mathrm{CT}$ & $\mathrm{TT}$ \\
\hline ת1 & 0,59 & 0,41 & 0,24 & 0,70 & 0,06 \\
\hline RYR1-20298 & A & G & $\mathrm{AA}$ & $A G$ & GG \\
\hline 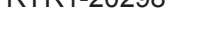 & 0,56 & 0,44 & 0,34 & 0,46 & 0,21 \\
\hline RYR1-21643* & C & $\mathrm{T}$ & $\mathrm{CC}$ & $\mathrm{CT}$ & TT \\
\hline 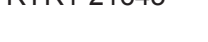 & 0,94 & 0,06 & 0,87 & 0,13 & 0 \\
\hline RYR1-26680 & $A$ & $C$ & AA & $A C$ & $\mathrm{CC}$ \\
\hline 促 & 0,87 & 0,13 & 0,73 & 0,27 & 0 \\
\hline RYR1-27871 & $C$ & $\mathrm{~T}$ & $\mathrm{CC}$ & $\mathrm{CT}$ & TT \\
\hline 19 & 0,76 & 0,24 & 0,74 & 0,05 & 0,21 \\
\hline RYR1-27970 & $\mathrm{C}$ & $\mathrm{T}$ & $\mathrm{CC}$ & $\mathrm{CT}$ & $\mathrm{TT}$ \\
\hline & 0,90 & 0,10 & 0,80 & 0,18 & 0,01 \\
\hline RYR1-27982 & C & $\mathrm{T}$ & CC & $\mathrm{CT}$ & $\mathrm{TT}$ \\
\hline & 0,86 & 0,14 & 0,74 & 0,24 & 0,02 \\
\hline RYR1-28046* & C & G & $\mathrm{CC}$ & CG & GG \\
\hline तालि-20040 & 0,99 & 0,01 & 0,99 & 0,01 & 0 \\
\hline RYR1-28068* & $\mathrm{C}$ & $\mathrm{T}$ & CC & $\mathrm{CT}$ & TT \\
\hline 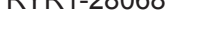 & 1,00 & 0,00 & 0,99 & 0,01 & 0 \\
\hline
\end{tabular}
marcador (Allelic and genotypic frequencies in each marker).

*Marcadores eliminados de la asociación fenotipo-genotipo por baja frecuencia alélica o genotípica. 
Tabla IV. Asociación según marcador (Associated markers).

\begin{tabular}{|c|c|c|c|c|c|c|c|c|c|c|c|}
\hline \multirow{2}{*}{ Marcador } & \multirow{2}{*}{ Parámetro } & \multirow{2}{*}{ Músculo } & & \multicolumn{7}{|c|}{ Comparaciones } & \multirow[b]{2}{*}{ SE } \\
\hline & & & & Promedio & SE & & Promedio & SE & & Promedio & \\
\hline CAST2959 & CRAr & LD & $\mathrm{AA}$ & $33,03^{a}$ & 7,49 & $A G$ & $31,56^{b}$ & 6,67 & GG & $31,46^{b}$ & 6,2 \\
\hline RYR1-11195 & CRAc & LD & AA & $18,18^{a}$ & 6,55 & $A G$ & $21,98^{b}$ & 6,89 & GG & $22,34^{b}$ & 7,86 \\
\hline
\end{tabular}

Valores promedio en negrita indican el valor deseable según el parámetro de CRA analizado. Valores con diferente superíndice en una misma fila indican diferencia $(p<0,001)$.

Casas et al. (2006), Van Eenennaam et al. (2007), Allais et al. (2011) y Li et al. (2010) encontraron que los individuos con genotipo AA presentaban carne más blanda respecto a animales AG y GG. Lo anterior evidencia claramente la relación existente entre la proteólisis autógena del músculo y la retención del agua en la carne durante su proceso de maduración, lo que genera una matriz proteína-agua mucho más estable limitando la pérdida de agua propia del músculo-carne durante el proceso de maduración y el almacenamiento en el postmortem. Previamente, Leal y Jiménez (2015) establecieron diferencias in-silico en la estabilidad molecular del ARNm del alelo G del marcador CAST2959, cuando se comparó con el del alelo A. Teóricamente las diferencias en estabilidad podrían suministrar al ARNm del alelo $G$ una mayor estabilidad, permitiendo una mayor expresión de la proteína CAST y por lo tanto una mayor tasa de inhibición de la proteína CAPN1, lo que originaría carnes más duras (Casas et al. (2006), Van Eenennaam et al. (2007), Allais et al. (2011) y Li et al. (2010) y con mayores pérdidas de agua en carne cruda para animales que posean genotipos AG y GG.

Otros marcadores del sistema CAPN1-CAST (UoGCAST, CAST1, CAPN530, CAPN4751 y CAST2870), reportados en la literatura con asociación a parámetros de terneza de la carne, fueron evaluados en el presente estudio pero no se encontraron asociados con los parámetros de CRA evaluados (Schenkel et al., 2006; Li et al., 2010; Reardon et al., 2010; Pinto et al., 2010; Schenkel et al., 2006; Page et al., 2002; Corva et al., 2007; Van Eenennaam et al., 2007; Casas et al., 2006). Es importante resaltar la existencia de diferencias entre razas y poblaciones, lo que puede limitar la validación de un mismo marcador y su asociación a un fenotipo determinado, dados los efectos ambientales e inclusive genéticos diferenciales, lo que destaca la importancia de realizar la validación de ellos en nuevas poblaciones objetivo.

\section{GEN RYR 1}

En el presente documento, se encontró asociación entre el polimorfismo RYR1-11195 y el parámetro de calidad cárnica capacidad de retención de agua en carne cocinada (CRAc) en el músculo LD, siendo los genotipos AG y GG los que presentan una mayor pérdida de peso asociada a una mayor pérdida de agua durante el sometimiento de la carne a la cocción, cuando son comparados con animales de genotipo AA. El polimorfismo RYR1-11195 corresponde a una mutación sinónima (rs41881999, http: / / www.ncbi.nlm.nih.gov / snp/?term=rs41881999) que codifica para el aminoácido prolina en la posición 362 de la proteína.

El proceso biológico mediante el cual esta mutación sinónima puede desencadenar un efecto fenotípico como el descrito en el presente documento, es complejo, dado que el efecto debe ser a nivel transcripcional en la molécula de ARNm de 15265 nucleótidos (http:/ / www.ensembl.org/Bos_taurus/Transcript/ Sequence_cDNA?db=core;g=ENSBTAG00000006999; $\mathrm{r}=18: 48502352-48631056$; $\mathrm{t}=$ ENSBTAT00000009228), lo cual sería difícil comprobar si el cambio de un nucleótido puede llegar a modificar la información análoga y / o digital de la molécula (Andrade, 2001) conduciendo a moléculas de ARNm menos estables y menos disponibles para su traducción. Lo anterior ha sido evidenciado tanto a nivel in-silico como a nivel in vitro en varios estudios previamente reportados (Duan et al., 2003; Johnson et al., 2011; Shen et al., 1999; Halvorsen et al., 2010; Leal y Jiménez 2014), los cuales han mostrado un posible mecanismo de regulación de la expresión causada por mutaciones sinónimas al mediar procesos de síntesis, maduración, transporte, traducción o degradación (Shen et al., 1999; Bartoszewski et al., 2010). Otro posible mecanismo de regulación corresponde al mediado por ARNs no traducidos con función reguladora de la expresión, en el cual el polimorfismo podría llegar a generar o eliminar un sitio de interacción molecular con el ARNm del RYR1 y por lo tanto modificar la expresión del gen (van Dijk et al., 2009).

Es importante resaltar que el gen RYR1 posee gran importancia en características asociadas a la CRA en el cerdo, pero ha sido pobremente evaluado en el bovino y el presente estudio lo resalta como de gran potencial en esta especie y posiblemente en el área de la producción animal en general.

\section{GEN PRKAG3}

Los marcadores evaluados del gen PRKAG3 no presentaron asociación fenotipo-genotipo en el presente estudio, sin embargo el marcador PRKAG3-2951 se encontró asociado con el parámetro $\mathrm{pH}$ a 24 horas y con la CRAc en lomo en el estudio de Reardon et al. (2010), siendo los animales con genotipo AG y AA los que presentaron un incremento en un $1 \%$ en su valor de pérdida de agua respecto a individuos con genotipo GG. Por lo anterior, se destaca la importancia de realizar procesos de validación de los marcadores en poblaciones no relacionadas con el fin de disminuir el reporte de falsos positivos en estudios de asociación que tengan como finalidad el uso de marcadores moleculares en procesos de selección animal.

\section{CONCLUSIONES}

Los marcadores CAST2959 (AA - genotipo benéfico) y RYR1-11195 (AA - genotipo benéfico) presentaron asociación con los parámetros CRAr y CRAc en el 
músculo Longissimus dorsi. Se destaca la importancia de estos dos marcadores moleculares en procesos de validación de polimorfismos con finalidades de selección y mejoramiento animal en Colombia.

\section{AGRADECIMIENTOS}

Al Ministerio de Agricultura de Colombia, al ICTA, a Colciencias y su Programa Jóvenes Investigadores e Innovadores y a la Dirección de Investigación sede Bogotá de la Universidad Nacional de Colombia.

\section{BIBLIOGRAFÍA}

Allais, S.; Journaux, L.; Levéziel, H.; Payet, N.; Raynaud, P.; Hocquette, J.; Lepetit, J.; Rousset, S.; Denoyelle, C.; Bernard, C. and Renand, G. 2011. Effects of polymorphisms in the calpastatin and $\mu$-calpain genes on meat tenderness in 3 French beef breeds. J Anim Sci, 89: 1-11.

Andrade, E. 2011. La dualidad análogo digital de la información se ejemplifica en el estudio de las moléculas de RNA. Acta Biol Colomb, 16: 15-42.

Barbanti, D. and Pasquini, M. 2005. Influence of cooking conditions on cooking loss and tenderness of raw and marinated chicken breast meat. LWT-Food Sci Technol, 38: 895-901.

Barendse, W.; Harrison, B.; Hawken, R.; Ferguson, D.; Thompson, J.; Thomas, M. and Bunch, R. 2007. Epistasis between calpain 1 and its inhibitor calpastatin within breeds of cattle. GSA, 176: 2601-2610.

Bartoszewski, R.; Jablonsky, M.; Bartoszewska, S.; Stevenson, L.; Dai, Q.; Kappes, J.; Collawn, J. and Bebok, Z. 2010. A synonymous single nucleotide polymorphism in F508 CFTR alters the secondary structure of the mRNA and the expression of the mutant protein. J Biol Chem, 285: 28741-28748.

Bastos, E.; Cravador, A.; Azevedo, J. and Guedes, E. 2001. Single strand conformation polymorphism (SSCP) detection in six genes in Portuguese indigenous sheep breed Churra da Terra Quente. Biotechnol Agron Soc Environ, 5: 7-15.

Bee, G.; Anderson, A.; Lonergan, S. and Huff-Lonergan, E. 2007. Rate and extent of $\mathrm{pH}$ decline affect proteolysis of cytoskeletal proteins and water-holding capacity in pork. Meat Sci, 76: 359-365.

Bertram, H.; Andersen, H.; Karlsson, A.; Horn, P.; Hedegaard, J.; Nørgaard, L. and Engelsen, S. 2003. Prediction of technological quality (cooking loss and Napole Yield) of pork based on fresh meat characteristics. Meat Sci, 65: 707-712.

Brunner, R.; Srikanchai, T.; Murani, E.; Wimmers, K. and Ponsuksili, S. 2012. Genes with expression levels correlating to drip loss prove association of their polymorphism with water holding capacity of pork. Mol Biol Rep, 39: 97-107.

Café, L.; Mclntyre, B.; Robinson, D.; Geesink, G.; Barendse, W.; Pethick, D.; Thompson, J. and Greenwood, P. 2010. Production and processing studies on calpain-system gene markers for tenderness in Brahman cattle: 2. Objective meat quality. J Anim Sci, 88: 3059-3069.

Casas, E.; White, S.; Wheeler, T.; Shackelford, S.; Koohmaraie, M.; Riley, D.; Chase, C.; Johnson, D. and Smith, T. 2006. Effects of calpastatin and $\mu$-calpain markers in beef cattle on tenderness traits. J Anim Sci, 84: $520-525$

Casas, E.; White, S.; Riley, D.; Smith, T.; Brenneman, R.; Olson, T.; Johnson, D.; Coleman, S.; Bennett, G. and Chase, C. 2005. Assessment of single nucleotide polymorphisms in genes residing on chromosomes 14 and 29 for association with carcass composition traits in Bos indicus cattle. J Anim Sci, 83: 13-19.

Chung, H. and Davis, M. 2011 . Effects of calpain genotypes on meat tenderness and carcass traits of Angus bulls. Mol Biol Rep, 38: 4575-4581.

Chung, H.; Davis, M. and Hines, H. 2000. Genetic variants detected by PCR-RFLP in intron 6 of the bovine calpastatin gene. Department of Animal Sciences. The Ohio State University. Columbus. USA.

Corva, P.; Soria, L.; Schor, A.; Villarreal, E.; Pérez, M.; Motter, M.; Mezzadra, C.; Melucci, L.; Miquel, C.; Paván, E.; Depetris, G.; San- tini, F. and Grigera, J. 2007. Association of CAPN1 and CAST gene polymorphisms with meat tenderness in Bos taurus beef cattle from Argentina. Genet Mol Biol, 30: 1064-1069.

Cui, J.; Du, H.; Liang, Y.; Deng, X.; Li, N. and Zhang, X. 2006. Association of polymorphisms in the promoter region of chicken prolactin with egg production. Poult Sci, 85: 26-31.

Curi, R.; Chardulo, J.; Giusti, J.; Silveira, A.; Martins, C. and de Oliveira, H. 2010. Assessment of GH1, CAPNI and CAST polymorphisms as markers of carcass and meat traits in Bos indicus and Bos taurus-Bos indicus cross beef cattle. Meat Sci, 86: 915-920.

Duan, J., Wainwright, M., Comeron, J., Saitou, N., Sanders, A., Gelernter, J. and Gejman, P., 2003. Synonymous mutations in the human dopamine receptor D2 (DRD2) affect mRNA stability and synthesis of the receptor. Hum Mol Gen, 12: 205-216.

Enfält, A.; von Seth, G.; Josell, A.; Lindahl, G.; Hedebro, I.; Braunschweig, M.; Andersson, L. and Lundström, K. 2006. Effects of a second mutant allele (V1991) at the PRKAG3 (RN) locus on carcass composition in pigs. Livest Sci, 99: 131-139.

Fessenden, J.; Feng, W.; Pessah, I. and Allen, P. 2004. Mutational analysis of putative calcium binding motifs within the skeletal ryanodine receptor isoform, RyR1. J Biol Chem, 279: 53028-53035.

Grahame, D. 2005. New roles for the LKB1 AMPK pathway. Curr Opin Cell Biol, 17: 167-173.

Halvorsen, M.; Martin, J.; Broadaway, S. and Laederach, A., 2010. Disease-associated mutations that alter the RNA structural ensemble. PLoS Genetics, 6.

Johnson, A.; Trumbower, H. and Sadee, W. 2011 . RNA structures affected by single nucleotide polymorphisms in transcribed regions of the human genome. Bioinformatics, $2.30 \mathrm{pp}$. http://www.webmedcentral.com/ article_view/1600 (13/3/2015).

Lahucky, R.; Christian, L.; Kovaq, L.; Staldert, J. and Baverova, M. 1997. Meat quality assessed ante- and post mortem by different ryanodine receptor gene status of pigs. Meat Sci, 41: 211-285.

Leal, J. and Jiménez, L. 2014. Análisis in-silico de mutaciones puntuales en el gen PRKAG3 bovino asociadas a calidad cárnica. Arch Zootec, 63: 121-132.

Leal, J. y Jiménez, L. 2015. Análisis computacional del efecto de mutaciones puntuales en los genes del sistema $\mu$-calpaína y calpastatina en calidad de carne bovina. Rev Med Vet Zoot, 62.

Leal, J.; Jiménez, L.; Ariza, M.; Manrique, C.; López, J.; Martínez, C.; Pinilla, Y.; Ríos, M.; Ortiz, Y. y Jiménez, A. 2014. Efecto del tipo genético y la maduración sobre la retención de agua en carne de toros castrados. Arch. Zootec, 63: 409-418.

Lee, Y.; Kwon, E.; Cho, E.; Park, D.; Kim, B.; Park, H.; Park, B.; Jang, I.; Choi, J.; Bang, W. and Kim, C. 2011. Association analysis of polymorphism in KIAA1717, HUMMLC2B, DECR1 and FTO genes with meat quality traits of the Berkshire breed. Afr J Biotechnol, 10: 5068-5074.

Li, J.; Zhang, L.; Gan, Q.; Li, J.; Gao, H.; Yuan, Z.; Gao, X.; Chen, J. and $\mathrm{Xu}, \mathrm{Z}$. 2010. Association of CAST gene polymorphisms with carcass and meat quality traits in chinese commercial cattle herds. Asian-Australas J Anim Sci, 23: 1405-1411.

Lindahl, G.; Enält, A.; Von Seth, G.; Josell, A.; Hedebro, I.; Andersen, H.; Braunschweig, M.; Andersson, L.; Lundstöm, K. 2004a. A second mutant allele (V199I) at the PRKAG3 (RN) locus-II. Effect on colour characteristics of pork loin. Meat Sci, 66: 621-627.

Lindahl, G.; Enält, A.; Von Seth, G.; Josell, A.; Hedebro, I.; Andersen, H.; Braunschweig, M.; Andersson, L. and Lundstöm, K. 2004b. A second mutantallele (V1991) at the PRKAG3 (RN) locus-Effect on technological meat quality of pork loin. Meat Sci, 66: 609-619.

Martínez, C.; Manrique, C.; Elzo, M. and Jiménez, A. 2012. Additive genetic group and heterosis effects on growth and corporal composition of crossbred cattle in southern Cesar (Colombia). Rev Colomb Cienc Pec, 25: 377-390.

Otto, G.; Roehe, R.; Looft, H.; Thoelking, L.; Knap, P.; Rothschild, M.; Plastow, G. and Kalm, E. 2007. Associations ofDNA markers with meat quality traits in pigs with emphasis on drip loss. MeatSci, 75: 185-195. 
Page, B.; Casas, E.; Heaton, M.; Cullen, N.; Hyndman, D.; Morris, C.; Crawford, A.; Wheeler, T.; Koohmaraie, M.; Keele, J. and Smith, T. 2002. Evaluation of single-nucleotide polymorphisms in CAPN1 for association with meat tenderness in cattle. J Anim Sci, 80: 3077-3085.

Pinto, L.; Ferraz, J.; Meirelles, F.; Eler, J.; Rezende, F.; Carvalho, M.; Almeida, H. and Silva, R. 2010. Association of SNPs on CAPN1 and CAST genes with tenderness in Nellore cattle. Gen Mol Res, 9: 1431-1442.

Reardon, W.; Mullen, A.; Sweeney, T. and Hamill, R. 2010. Association of polymorphisms in candidate genes with colour, water-holding capacity, and composition traits in bovine M. longissimus and $M$. semimembranosus. Meat Sci, 86: 270-275.

Schenkel, F.; Miller, S.; Jiang, Z.; Mandell, I.; Ye, X.; Li, H. and Wilton, J. 2006. Association of a single nucleotide polymorphism in the calpastatin gene with carcass and meat quality traits of beef cattle. $J$ Anim Sci, 84: 291-299.

Shen, L.; Basilion, J. and Stanton, V. 1999. Single-nucleotide polymorphisms can cause different structural folds of mRNA. PNAS, 96: 7871-7876.

Škrlep, M.; Candek, M.; Kavar, T.; Žlender, B.; Hortós, M.; Gou, P.; Arnau, J.; Evans, G.; Southwood, O.; Diestre, A.; Robert, N.; Dutertre, C. and Santé, V. 2010a. Association of PRKAG3 and CAST genetic polymorphisms with traits of interest in dry-cured ham production: Comparative study in France, Slovenia and Spain. LivestSci, 128: 60-66.

Škrlep, M.; Kavar, T. and Candek, M. 2010b. Comparison of PRKAG3 and RYR1 gene effect on carcass traits and meat quality in Slovenian commercial pigs. Czech J Anim Sci, 55: 149-159.

Straadt, I.; Rasmussen, M.; Andersen, H. and Bertram, H. 2007. Aginginduced changes in microstructure and water distribution in fresh and cooked pork in relation to water-holding capacity and cooking loss-A combined confocal laser scanning microscopy (CLSM) and low-field nuclear magnetic resonance relaxation study. Meat Sci, 75: 687-695.

Sunnucks, P.; Wilson, A.; Beheregaray, L.; Zenger, K.; French, J. and Taylor, A. 2000. SSCP is not so difficult: the application and utility of single-stranded conformation polymorphism in evolutionary biology and molecular ecology. Mol Ecol, 9: 1699-1710.

Van Dijk, S.; Dooijes, D.; dos Remedios, C.; Michels, M.; Lamers, J.; Winegrad, S.; Schlossarek, S.; Carrier, L.; Ten Cate, F.; Stienen, G. and van der Velden, J. 2009. Cardiac myosin-binding protein C mutations and hypertrophic cardiomyopathy haploinsufficiency, deranged phosphorylation, and cardiomyocyte dysfunction. Circulation, 119: 1473-1483.

Van Eenennaam, A.; Li, J.; Thallman, R.; Quaas, R.; Dikeman, M.; Gill, C.; Franke, D. and Thomas, M. 2007. Validation of commercial DNA tests for quantitative beef quality traits. J. Anim Sci, 85: 891-900.

Van Oeckel, M.; Warnants, N. and Boucque, V. 1999. Comparison of different methods for measuring water holding capacity and juiciness of pork versus on-line screening methods. Meat Sci, 51: 313-320.

White, S.; Casas, E.; Wheeler, T.; Shackelford, S.; Koohmaraie, M.; Riley, D.; Chase, C.; Johnson, D.; Keele, J. and Smith, T. 2005. A new single nucleotide polymorphism in CAPN1 extends the current tenderness marker test to include cattle of Bos indicus, Bos taurus, and crossbred descent. J Anim Sci, 83: 2001-2008.

www.ensembl.org/Bos_taurus/Transcript/Sequence_cD NA? $\mathrm{db}=$ core $; g=$ ENSBTAG00000006999; $r=18: 48502352$ 48631056; $t=$ ENSBTATO0000009228 (13/3/2015).

www.ncbi.nlm.nih.gov/snp/?term=rs41881999 (13/3/2015).

Yu, S.; Kim, J.; Chung, H.; Jung, K.; Lee, Y.; Yoon, D.; Lee, S.; Choi, I.; Bottema, C.; Sang, B. and Lee, J. 2005. Molecular cloning and characterization of bovine PRKAG3 gene: structure, expression and single nucleotide polymorphism detection. JAnim Breed Genet, 122: 294-301.

Zhang, W.; Lonergan, S.; Gardner, M. and Huff-Lonergan, E. 2006. Contribution of postmortem changes of integrin, desmin and $\mu$-calpain to variation in water holding capacity of pork. Meat Sci, 74: 578-585.

Zhang, W.; Lonergan, S.; Gardner, M. and Huff-Lonergan, E. 2006. Contribution of postmortem changes of integrin, desmin and $\mu$-calpain to variation in water holding capacity of pork. Meat Sci, 74: 578-585.

Zhou, G.; Cao, Y.; Li, M.; Zhang, L.; Yu, Y. and Jin, H. 2010. Meat quality and carcass traits in relation to HGD-BstXI and HGD-Haell PCR-RFLP polymorphism in Chinese red cattle. Meat Sci, 85: 270-273.

Zhou, G.; Cao, Y.; Li, M.; Zhang, L.; Yu, Y. and Jin, H. 2010. Meat quality and carcass traits in relation to HGD-BstXI and HGD-Haell PCR-RFLP polymorphism in Chinese red cattle. Meat Sci, 85: 270-273. 